\title{
Hierarchical Mixed Hybridized Methods for Elliptic Problems
}

\author{
Paola Causin \\ Dipartimento di Matematica "F. Enriques" \\ Università degli Studi di Milano \\ via Saldini 50, 20133 Milano, Italy \\ Riccardo Sacco * \\ Dipartimento di Matematica "F. Brioschi" \\ Politecnico di Milano \\ via Bonardi 9, 20133 Milano, Italy
}

\begin{abstract}
In this article, we propose a computational procedure for the efficient implementation of Dual Mixed Hybridized methods of arbitrary degree. Three main mathematical tools are used: $i$ ) hierarchical finite element bases to yield a vertical splitting (completion) of each approximation space into a lower degree and a defect correction part; ii) the Helmholtz decomposition principle for the vector-valued variable to yield an horizontal splitting of the defect correction part into solenoidal and non-solenoidal subspaces; iii) fixed-point iterations to solve the two classes of approximate decoupled subproblems resulting from $i$ ) and $i i$ ), one of generalized displacement type (upon static condensation) and the other of local saddle-point type. Exploiting the equivalence between hierarchical completion and Variational Multiscale Modeling, we also devise and numerically validate a hierarchical a-posteriori error estimator for Dual Mixed methods in hybridized form.
\end{abstract}

Key words: mixed finite element methods, hybridization techniques, hierarchical basis, elliptic boundary value problems.

\footnotetext{
* Corresponding author:

Email address: riccardo.sacco@polimi.it (Riccardo Sacco).
} 


\section{Introduction}

In this work, we are concerned with a computational procedure for dealing with Dual Mixed Hybridized (DMH) methods applied to the numerical discretization of the following elliptic model problem:

Given $f \in L^{2}(\Omega)$ and $g_{D} \in H^{1 / 2}(\Gamma)$, find $(\boldsymbol{q}, u)$ such that

$$
\begin{aligned}
\boldsymbol{q}+\kappa \nabla u=0 & \text { in } \Omega, \\
\operatorname{div} \boldsymbol{q}+\sigma u=f & \text { in } \Omega, \\
u=g_{D} & \text { on } \Gamma,
\end{aligned}
$$

where $\Omega \subset \mathbb{R}^{2}$ is a polygonal domain with boundary $\Gamma, \kappa(\boldsymbol{x})$ is a bounded diffusion coefficient such that $\kappa(\boldsymbol{x}) \geq \kappa_{0}>0$ a.e. in $\Omega$, and $\sigma(\boldsymbol{x})$ is a reaction coefficient such that $\sigma(\boldsymbol{x}) \geq 0$ a.e. in $\Omega$.

Dual Mixed (DM) methods provide an accurate representation of both primal and dual variables (this latter representing the flux). However, it is well known that DM methods give rise to nondefinite linear algebraic systems of large size, the solution of which is computationally expensive [4]. Resorting to hybridization [2] yields a symmetric definite positive of reduced size for the sole hybrid variable (Lagrange multiplier of the interelement continuity of the normal flux), which exhibits superconvergence properties and provides a more accurate representation of the solution. Despite such clear advantages, only a few papers in the literature deal with DM methods in their hybridized version. Two relevant, and recent, contributions are given in $[9,10]$, where a variational characterization of the hybridization procedure provides a systematic tool for the computation of local matrices and error analysis with respect to approximations of arbitrary polynomial degree. Efficient multilevel iterative solvers for DMH methods have been investigated in [16], where hybridized Raviart-Thomas (RT) elements of lowest degree are analyzed by exploiting their equivalence with the nonconforming displacement-based formulation of Crouzeix-Raviart type $[11,22]$. Again in the context of multilevel approaches, the theoretical foundation of DMH methods of [9] is exploited in [14] to devise a uniformly well conditioned Schwarz preconditioner for approximations of arbitrary polynomial degree. Eventually, in the area of a-posteriori error estimators for DMH formulations, we mention the works [5,6,24], which deal, using different approches, with the lowest order degree method, relying on the connection with nonconforming approximations.

Here, we consider DMH methods of arbitrary degree for the numerical approximation of (1). With this aim, we first decompose the finite element spaces for 
scalar and vector-valued variables "vertically" into a $p$-type hierarchy using the completion approach of [19], which allows to generate a basis of degree $k+1$ as a defect correction to the basis of degree $k$. Then, as proposed in $[15,17,24]$ in the field of a-posteriori error estimators for DM formulations, we additionally decompose "horizontally" the defect correction space for the flux variable via a strategy based on the Helmholtz principle [13]. We notice that the completion concept of [19] has been previously applied in [1], where an a-posteriori error estimator for DM methods is devised and an explicit computation of the local hierarchical basis functions is carried out in the case of RT elements of degree 1 . In this article, instead, we apply the vertical-horizontal decomposition strategy directly to compute the solution of the DMH problem. This allows to single out a systematic substructuring which naturally lends itself to an efficient approximate block iterative implementation. The above introduced completion concept can be reinterpreted as an instance of Variational Multiscale Modeling [18], recently applied to the Discontinuous Petrov-Galerkin setting in [3], and of Enrichment Methods [12]. This reinterpretation allows to devise an a-posteriori error estimator for the DMH method, based on the use of the defect correction part as an indicator of error with respect to the lower degree approximation.

The remainder of the paper is organized as follows. In Sect. 2, the DM and DMH methods are recalled along with the mathematical notation. In Sect. 3, the hierarchical representation and Helmholtz decomposition of finite element spaces are introduced. Details are provided for the construction of hierarchical local basis functions for the flux variable in the case of RT finite element spaces of arbitrary order $k \geq 0$ and a practical example of computation is provided in the case $k=1$. In Sect. 4 , the hierarchical basis functions are used in the DMH problem and approximate versions of the flux mass matrix are investigated in order to decouple the scales of the problem. A fixed point iterative procedure is then proposed to efficiently implement the approximate DMH formulation. In Sect. 5, the techniques of [9] are extended to obtain the entries of the coefficient matrix and of the right-hand side of the condensed system of the approximate DMH formulation. In Sect. 6, a hierarchical a-posteriori error estimator, that can be cheaply implemented in the iterative procedure, is introduced and numerically validated. In Sect. 7, some concluding remarks are drawn and future perspectives are indicated.

\section{Mixed and Mixed-Hybridized Discretizations}

For $S \subseteq \Omega$, we introduce the Hilbert ansatz space

$$
H(\operatorname{div} ; S):=\left\{\boldsymbol{v} \in\left(L^{2}(S)\right)^{2} \mid \operatorname{div} \boldsymbol{v} \in L^{2}(S)\right\},
$$


equipped with the inner product

$$
(\boldsymbol{v}, \boldsymbol{q})_{H(\operatorname{div} ; S)}:=(\boldsymbol{v}, \boldsymbol{q})_{0 ; S}+(\operatorname{div} \boldsymbol{v}, \operatorname{div} \boldsymbol{q})_{0 ; S},
$$

$(\cdot, \cdot)_{0 ; S}$ being the standard $L^{2}$ product on $S$. The associated graph norm is $\|\boldsymbol{v}\|_{\text {div; } S}=(\boldsymbol{v}, \boldsymbol{v})_{\operatorname{div} ; S}^{1 / 2}$. The mixed weak formulation of $(1)$ reads:

Find $(\boldsymbol{q}, u) \in\left(H(\operatorname{div} ; \Omega) \times L^{2}(\Omega)\right)$ such that for all $(\boldsymbol{v}, w) \in(H(\operatorname{div} ; \Omega) \times$ $\left.L^{2}(\Omega)\right)$

$$
\begin{aligned}
a(\boldsymbol{q}, \boldsymbol{v})+b(\boldsymbol{v}, u) & =\mathcal{G}(\boldsymbol{v}), \\
b(\boldsymbol{q}, w)+d(u, w) & =\mathcal{F}(w),
\end{aligned}
$$

where the bilinear forms $a(\cdot, \cdot), b(\cdot, \cdot), c(\cdot, \cdot)$ and the linear forms $\mathcal{F}(\cdot)$ and $\mathcal{G}(\cdot)$ are defined as

$$
\begin{array}{rlrl}
a(\boldsymbol{q}, \boldsymbol{v}) & =\int_{\Omega} \kappa^{-1} \boldsymbol{q} \cdot \boldsymbol{v} d x, & \boldsymbol{q}, \boldsymbol{v} \in H(\operatorname{div} ; \Omega), \\
b(\boldsymbol{v}, w) & =-\int_{\Omega} w \operatorname{div} \boldsymbol{v} d x, & \boldsymbol{v} \in H(\operatorname{div} ; \Omega), w \in L^{2}(\Omega), \\
d(u, w) & =-\int_{\Omega} \sigma u w d x, & & u, w \in L^{2}(\Omega), \\
\mathcal{F}(w) & =-\int_{\Omega} f w d x, & & w \in L^{2}(\Omega), \\
\mathcal{G}(\boldsymbol{v}) & =-\int_{\Gamma} g_{D} \boldsymbol{v} \cdot \boldsymbol{n} d s, & \boldsymbol{v} \in H(\operatorname{div} ; \Omega) .
\end{array}
$$

Associated with the bilinear form $a(\cdot, \cdot)$, we also define the energy norm

$$
\|\boldsymbol{v}\|_{\operatorname{div} ; S}:=\left(\int_{S} \kappa^{-1} \boldsymbol{v} \cdot \boldsymbol{v} d x+\int_{S} \operatorname{div} \boldsymbol{v} \cdot \operatorname{div} \boldsymbol{v} d x\right)^{1 / 2} .
$$

In view of the finite element discretization of (2), we let $\bar{\Omega}=\bigcup \bar{K}$ be a regular [8] partition $\mathcal{T}_{h}$ of the domain $\Omega$ into triangular elements $K$. We denote by $\partial K$ and $\boldsymbol{n}$ the boundary of the element and its outward unit normal vector (according to a counterclockwise orientation along $\partial K$ ), respectively. Moreover, we denote by $\mathcal{E}_{h}$ the set of all the edges of $\mathcal{T}_{h}$, and by $\mathcal{E}_{h, i}$ the set of the internal edges of $\mathcal{E}_{h}$, and we denote by $\mathrm{Ni}$ the number of internal edges of the mesh. For $k \geq 0$, we let $\mathbb{P}_{k}(K)$ be the local space of polynomials in two variables of total degree at most $k$ on $K$, and by $W_{k}\left(\Omega ; \mathcal{T}_{h}\right):=\prod_{K \in \mathcal{T}_{h}} \mathbb{P}_{k}(K)$ the corresponding global space. Furthermore, we let $\mathbb{R}_{k}(K)$ be the Raviart-Thomas finite element space [21] of degree $k$ on $K$, defined as

$$
\mathbb{R} \mathbb{T}_{k}(K):=\left(\mathbb{P}_{k}(K)\right)^{2} \oplus \mathcal{P}_{k}(K) \boldsymbol{x},
$$

where $\boldsymbol{x}=(x, y)^{t}$ and $\mathcal{P}_{k}(K):=\operatorname{span}\left\{x^{\alpha} y^{\beta}, \alpha+\beta=k\right\}$. Notice that if a function $\left.\boldsymbol{v}\right|_{K}$ belongs to $\mathbb{R T}_{k}(K)$, then $\left.\operatorname{div} \boldsymbol{v}\right|_{K}$ belongs to $\mathbb{P}_{k}(K)$ and $\left.\boldsymbol{v} \cdot \boldsymbol{n}\right|_{\partial K}$ 
belongs to $R_{k}(\partial K)$. We denote by $\boldsymbol{V}_{k}\left(\Omega ; \mathcal{T}_{h}\right):=\prod_{K \in \mathcal{T}_{h}} \mathbb{R T}_{k}(K)$ the global space corresponding to (5). Finally, we set

$$
\mathbb{R} \mathbb{T}_{k}\left(\Omega ; \mathcal{T}_{h}\right):=\left\{\boldsymbol{v} \in H(\operatorname{div} ; \Omega)|\boldsymbol{v}|_{K} \in \mathbb{R}_{k}(K)\right\}
$$

Having introduced the above spaces, we can state the following DM finite element approximation of problem (1):

Find $\left(\boldsymbol{q}_{k}, u_{k}\right) \in\left(\mathbb{R} \mathbb{T}_{k}\left(\Omega ; \mathcal{T}_{h}\right) \times W_{k}\left(\Omega ; \mathcal{T}_{h}\right)\right)$ such that for all $(\boldsymbol{v}, w) \in\left(\mathbb{R} \mathbb{T}_{k}\left(\Omega ; \mathcal{T}_{h}\right) \times\right.$ $\left.W_{k}\left(\Omega ; \mathcal{T}_{h}\right)\right)$

$$
\begin{aligned}
a\left(\boldsymbol{q}_{k}, \boldsymbol{v}\right)+b\left(\boldsymbol{v}, u_{k}\right) & =\mathcal{G}(\boldsymbol{v}), \\
b\left(\boldsymbol{q}_{k}, w\right)+d\left(u_{k}, w\right) & =\mathcal{F}(w) .
\end{aligned}
$$

The hybridization procedure makes use in the previous system of an additional dependent variable to relax the interelement continuity for the normal component of the flux [2]. The formulation is based on the introduction of the space of single-valued functions on the internal edges of the triangulation

$$
L_{k}\left(\mathcal{E}_{h, i}\right)=\left\{\eta \in L^{2}\left(\mathcal{E}_{h, i}\right)|\eta|_{\partial K} \in R(\partial K), \forall K \in \mathcal{T}_{h}, \partial K \cap \Gamma=\emptyset\right\}
$$

where we let $R_{k}(\partial K)$ be the space of polynomials in one variable of total degree at most $k$ on each edge of $\partial K$. Observe that functions belonging to $R_{k}(\partial K)$ are not necessarily continuous at the vertices of $K$. Then, the DMH formulation of (1) reads:

Find $\left(\boldsymbol{q}_{k}, u_{k}, \lambda_{k}\right) \in\left(\mathbf{V}_{k}\left(\Omega ; \mathcal{T}_{h}\right) \times W_{k}\left(\Omega ; \mathcal{T}_{h}\right) \times L_{k}\left(\mathcal{E}_{h, i}\right)\right)$ such that for all $(\boldsymbol{v}, w, \eta) \in\left(\mathbf{V}_{k}\left(\Omega ; \mathcal{T}_{h}\right) \times W_{k}\left(\Omega ; \mathcal{T}_{h}\right) \times L_{k}\left(\mathcal{E}_{h, i}\right)\right)$

$$
\begin{aligned}
\sum_{K \in \mathcal{T}_{h}} a^{K}\left(\boldsymbol{q}_{k}, \boldsymbol{v}\right)+\sum_{K \in \mathcal{T}_{h}} b^{K}\left(\boldsymbol{v}, u_{k}\right)+\sum_{K \in \mathcal{T}_{h}} c^{K}\left(\boldsymbol{v}, \lambda_{k}\right) & =\mathcal{G}(\boldsymbol{v}), \\
\sum_{K \in \mathcal{T}_{h}} b^{K}\left(\boldsymbol{q}_{k}, w\right)+\sum_{K \in \mathcal{T}_{h}} d^{K}\left(u_{k}, w\right) & =\mathcal{F}(w), \\
\sum_{K \in \mathcal{T}_{h}} c^{K}\left(\boldsymbol{q}_{k}, \eta\right) & =0,
\end{aligned}
$$

where the bilinear forms $a^{K}(\cdot, \cdot), b^{K}(\cdot, \cdot), d^{K}(\cdot, \cdot)$ are the localization on $K$ of the corresponding bilinear forms of (3) and where we have introduced the bilinear form

$$
c^{K}(\boldsymbol{v}, \eta)=\int_{\partial K} \eta \boldsymbol{v} \cdot \boldsymbol{n} d s, \quad \boldsymbol{v} \in \mathbb{R}_{k}(K), \eta \in R_{k}(\partial K), \partial K \cap \Gamma=\emptyset .
$$

Equation $(9)_{3}$ expresses the fact that functions in $\mathbf{V}_{k}\left(\Omega ; \mathcal{T}_{h}\right)$, that a-priori have discontinuous normal components over $\mathcal{E}_{h, i}$, satisfy in weak form an interele- 
ment compatibility condition, which physically corresponds to the actionreaction principle. Problem (9) admits a unique solution (see [4] for a proof).

Let us define now the operators $\mathcal{A}: \mathbf{V}_{k}\left(\Omega ; \mathcal{T}_{h}\right) \mapsto \mathbf{V}_{k}\left(\Omega ; \mathcal{T}_{h}\right)^{\prime}, \mathcal{B}: \mathbf{V}_{k}\left(\Omega ; \mathcal{T}_{h}\right) \mapsto$ $W_{k}\left(\Omega ; \mathcal{T}_{h}\right)^{\prime}, \mathcal{C}: \mathbf{V}_{k}\left(\Omega ; \mathcal{T}_{h}\right) \mapsto L_{k}\left(\mathcal{E}_{h, i}\right)^{\prime}, \mathcal{D}: W_{k}\left(\Omega ; \mathcal{T}_{h}\right) \mapsto W_{k}\left(\Omega ; \mathcal{T}_{h}\right)^{\prime}$ by

$$
\begin{aligned}
& \left(\mathcal{A} \boldsymbol{q}_{k}, \boldsymbol{v}_{k}\right)_{\mathbf{V}_{k}\left(\Omega ; \mathcal{T}_{h}\right)}=a\left(\boldsymbol{q}_{k}, \boldsymbol{v}_{k}\right), \\
& \left(\mathcal{B} \boldsymbol{v}_{k}, w_{k}\right)_{W_{k}\left(\Omega ; \mathcal{T}_{h}\right)}=b\left(\boldsymbol{v}_{k}, w_{k}\right), \\
& \left(\mathcal{C} \boldsymbol{v}_{k}, \eta_{k}\right)_{L_{k}\left(\mathcal{E}_{h, i}\right)}=c\left(\boldsymbol{v}_{k}, \eta_{k}\right), \\
& \left(\mathcal{D} u_{k}, w_{k}\right)_{W_{k}\left(\Omega ; \mathcal{T}_{h}\right)}=d\left(u_{k}, w_{k}\right) .
\end{aligned}
$$

Then, the DMH problem can be stated as

$$
\left(\begin{array}{ccc}
\mathcal{A} & \mathcal{B}^{t} & \mathcal{C}^{t} \\
\mathcal{B} & \mathcal{D} & 0 \\
\mathcal{C} & 0 & 0
\end{array}\right)\left(\begin{array}{l}
\boldsymbol{q}_{k} \\
u_{k} \\
\lambda_{k}
\end{array}\right)=\left(\begin{array}{c}
\mathcal{G} \\
\mathcal{F} \\
0
\end{array}\right)
$$

where, with a slight abuse of notation, $\mathcal{G}$ and $\mathcal{F}$ denote here functionals on $\mathbf{V}_{k}\left(\Omega ; \mathcal{T}_{h}\right)$ and $W_{k}\left(\Omega ; \mathcal{T}_{h}\right)$, respectively. The variables $\boldsymbol{q}_{k}$ and $u_{k}$ can be statically eliminated from (11) to yield an equation in the sole hybrid variable $\lambda_{k}$

$$
\begin{aligned}
& \mathcal{A}^{-1}\left(\mathcal{A}+\mathcal{B}^{t}\left(\mathcal{D}-\mathcal{B} \mathcal{A}^{-1} \mathcal{B}^{t}\right)^{-1} \mathcal{B}\right) \mathcal{A}^{-1} C^{t} \lambda_{k}= \\
& \mathcal{C} \mathcal{A}^{-1}\left(\mathcal{A}+B^{t}\left(\mathcal{D}-\mathcal{B} \mathcal{A}^{-1} \mathcal{B}^{t}\right)^{-1} \mathcal{B}\right) \mathcal{A}^{-1} \mathcal{G}-\mathcal{C} \mathcal{A}^{-1} \mathcal{B}^{t}\left(\mathcal{D}-\mathcal{B} \mathcal{A}^{-1} \mathcal{B}^{t}\right)^{-1} \mathcal{F}
\end{aligned}
$$

Let $N_{\lambda}=(k+1) \mathrm{Ni}$ and $\left\{\lambda_{k}^{i}\right\}_{i=1}^{N_{\lambda}}$ be a basis for $L_{k}\left(\mathcal{E}_{h, i}\right)$, in such a way that $\lambda_{k}=\sum_{i=1}^{N_{\lambda}} \alpha_{i} \lambda_{k}^{i}$. Then, relation (12) can be written in matrix form as

$$
E \Lambda=H
$$

where $\Lambda=\left(\alpha_{i}\right)_{i=1, \ldots, N_{\lambda}}$ is the unknown vector, and the stiffness matrix $E$ and the right-hand side $H$ are computed from the matrices associated with the operators $\mathcal{A}, \mathcal{B}, \mathcal{C}, \mathcal{D}$ and $\mathcal{G}, \mathcal{F}$, respectively. The expression of the entries of $E$ and $H$ is given in Sect. 5 .

\section{Hierarchical-Helmholtz Decomposition of Approximation Spaces}

In this section, we introduce the two following mathematical tools: 
i) the hierarchical splitting of all the finite element spaces into lower order and defect correction contributions;

ii) the Helmholtz decomposition of the defect correction space for the flux variable.

Then, for a given nonnegative integer $k$, we let $X_{k}$ be a polynomial space of degree $\leq k$, and we construct the space of higher degree $X_{k+1}$ as the completion of $X_{k}$, i.e., by hierarchically enriching $X_{k}$ with the defect correction space $\widetilde{X}_{k+1}$ as

$$
X_{k+1}=X_{k} \oplus \widetilde{X}_{k+1} .
$$

Relation (14) represents a vertical decomposition of the considered finite element space, and corresponds to the splitting $i$ ). In the case where $X$ is a space of vector-valued functions, we also consider the Helmholtz decomposition

$$
X=X^{0} \oplus X^{\perp},
$$

where $(\cdot)^{0}$ indicates the divergence-free subset of $(\cdot)$ and $(\cdot)^{\perp}$ indicates its complement. Relation (15) represents a horizontal decomposition of the considered finite element space, and, setting $X=\widetilde{X}_{k+1}$, corresponds to the splitting $i i$ ).

\subsection{Hierarchical Basis Functions}

In this section, we construct the hierarchical representation of basis functions. The procedure for the scalar one-dimensional and two-dimensional spaces is an $L^{2}$ orthogonalization of basis functions in the defect space with respect to functions in the lower order space. The treatment of the Raviart-Thomas functions is more delicate. The theory developed in [19] allows to build a correction space of degree $k+1$, relying on the already existing bases of degree $k$. This same representation will be shown to provide also a straightforward characterization of the solenoidal part and of its complement in the defect space.

Applying (14) to $X_{k+1}=R_{k+1}(\partial K)$ yields the scalar edge hierarchical splitting

$$
R_{k+1}(\partial K)=R_{k}(\partial K) \oplus \widetilde{R}_{k+1}(\partial K)
$$

where

$$
\widetilde{R}_{k+1}(\partial K)=\left\{\widetilde{\eta} \in R_{k+1}(\partial K) \text { and } \int_{\partial K} \widetilde{\eta} \xi d s=0, \forall \xi \in R_{k}(\partial K), K \in \mathcal{T}_{h}\right\}
$$

It is easy to check that the restriction of $\widetilde{R}_{k+1}(\partial K)$ on each edge of $K$ consists of the Legendre polynomial of degree $k+1$ [23]. Applying (14) to $X_{k+1}=$ 


\begin{tabular}{cccccc}
\hline$k$ & space & $\operatorname{dim}$. completion & $\operatorname{dim} \Sigma_{A}$ & $\operatorname{dim} \Sigma_{B}$ & $\operatorname{dim} \Sigma_{C}$ \\
\hline 0 & $\mathbb{R T}_{1}$ & 5 & 3 & 2 & - \\
1 & $\mathbb{R T}_{2}$ & 7 & 3 & 3 & 1 \\
2 & $\mathbb{R T}_{3}$ & 9 & 3 & 4 & 2 \\
3 & $\mathbb{R T}_{4}$ & 11 & 3 & 5 & 3 \\
4 & $\mathbb{R T}_{5}$ & 13 & 3 & 6 & 4 \\
\hline
\end{tabular}

Table 1

Summary of the degrees of freedom of $\mathbb{R T}_{k+1}(K), k=0, \ldots, 4$, according to (20).

$\mathbb{P}_{k+1}(K)$ yields the scalar hierarchical splitting

$$
\mathbb{P}_{k+1}(K)=\mathbb{P}_{k}(K) \oplus \widetilde{\mathbb{P}}_{k+1}(K)
$$

where

$$
\widetilde{\mathbb{P}}_{k+1}(K)=\left\{\widetilde{w} \in \mathbb{P}_{k+1}(K) \text { and } \int_{K} \widetilde{w} \varphi d x=0, \forall \varphi \in \mathbb{P}_{k}(K), K \in \mathcal{T}_{h}\right\} .
$$

Applying (14) to $X_{k+1}=\mathbb{R T}_{k+1}(K)$ yields the vector hierarchical splitting

$$
\mathbb{R T}_{k+1}(K)=\mathbb{R T}_{k}(K) \oplus \widetilde{\mathbb{R T}}_{k+1}(K)
$$

We propose to characterize the completion space $\widetilde{\mathbb{R T}}_{k+1}(K)$ by the following set $\Sigma$ of degrees of freedom:

Type A (edge modes, $\Sigma_{A}$ ): $\quad \int_{\partial K} \boldsymbol{v} \cdot \boldsymbol{n} \widetilde{\eta} d s, \quad \widetilde{\eta} \in \widetilde{R}_{k+1}(\partial K), k \geq 0$,

Type B (internal modes, $\left.\Sigma_{B}\right): \quad \int_{K} \operatorname{div} \boldsymbol{v} \widetilde{\varphi} d x, \quad \widetilde{\varphi} \in \widetilde{\mathbb{P}}_{k+1}(K), \quad k \geq 0$,

Type C (internal modes, $\left.\Sigma_{C}\right): \quad \int_{K} \operatorname{curl} \boldsymbol{v} \widetilde{\alpha} d x, \quad \widetilde{\alpha} \in \widetilde{\mathbb{P}}_{k-1}(K), \quad k \geq 1$,

where $\boldsymbol{v} \in \widetilde{\mathbb{R T}}_{k+1}(K)$, and, for any $\mathbb{P} \in\left(H^{1}(K)\right)^{2}$, we define curl $\mathbb{P}:=\left(\frac{\partial p_{x}}{\partial y}-\right.$ $\left.\frac{\partial p_{y}}{\partial x}\right)$. Notice that $\operatorname{dim} \Sigma_{A}=3, \operatorname{dim} \Sigma_{B}=k+2$ and $\operatorname{dim} \Sigma_{C}=k$, from which it follows that $\operatorname{dim} \Sigma=\operatorname{dim} \Sigma_{A}+\operatorname{dim} \Sigma_{B}+\operatorname{dim} \Sigma_{C}=2 k+5$, which coincides with $\operatorname{dim} \widetilde{\mathbb{R T}}_{k+1}(K)=\operatorname{dim} \mathbb{R T}_{k+1}(K)-\operatorname{dim} \mathbb{R T}_{k}(K)$ (see Tab. 1).

Proposition 3.1 The finite element defined by the set $\Sigma$ is unisolvent.

Proof. Assume that the quantities $(20)_{1}-(20)_{3}$ are equal to zero, and prove that $\boldsymbol{v} \equiv \mathbf{0}$. Since $\left.\boldsymbol{v} \cdot \boldsymbol{n}\right|_{\partial K} \in \widetilde{R}_{k+1}(\partial K)$, the fact that $(20)_{1}$ is equal to zero implies that $\boldsymbol{v} \cdot \boldsymbol{n}=0$ on each edge of $\partial K$. Similarly, as $\operatorname{div} \boldsymbol{v} \in \widetilde{\mathbb{P}}_{k+1}(K)$, the 
fact that $(20)_{2}$ is equal to zero implies that $\operatorname{div} \boldsymbol{v}=0$, so that we can write $[4$, Corollary 3.1]

$$
\boldsymbol{v}=\operatorname{curl} \phi:=\left(-\frac{\partial \phi}{\partial y}, \frac{\partial \phi}{\partial x}\right)^{t}, \quad \phi \in \widetilde{\mathbb{P}}_{k+2}(K) .
$$

Let now $e \in \partial K$ be the edge connecting vertex $a$ to vertex $b$ (oriented from $a$ to $b$ ). Since

$$
0=\int_{e} \boldsymbol{v} \cdot \boldsymbol{n} d s=\phi(b)-\phi(a),
$$

we have that $\phi(a)=\phi(b)$. This implies that $\phi$ attains the same value $\bar{\phi}$ at each vertex of $K$. Without loss of generality, we set $\bar{\phi}=0$, and using (21) we get

$$
0=\int_{e} \boldsymbol{v} \cdot \boldsymbol{n} \widetilde{\eta}(s) d s=-\int_{e} \phi \frac{d \widetilde{\eta}(s)}{d s} d s, \quad \widetilde{\eta} \in \widetilde{P}_{k+1}(e) .
$$

The $k+1$ conditions of Eq. (22), together with the two conditions $\phi(a)=$ $\phi(b)=0$, imply that $\phi=0$ on $e$, and, as a consequence, $\left.\phi\right|_{\partial K}=0$. This latter property and the fact that $(20)_{3}$ is equal to zero gives $\int_{K} \triangle \phi \widetilde{\alpha} d x=0$ for all $\widetilde{\alpha} \in \widetilde{\mathbb{P}}_{k-1}(K)$, which implies that $\phi \equiv 0$ in $\bar{K}[19]$.

\subsection{Helmholtz Decomposition of Defect Correction Spaces}

Applying (15) to $X=\widetilde{\mathbb{R T}}_{k+1}(K)$ yields the Helmholtz splitting

$$
\widetilde{\mathbb{R T}}_{k+1}(K)=\widetilde{\mathbb{R T}}_{k+1}^{0}(K) \oplus \widetilde{\mathbb{R T}}_{k+1}^{\perp}(K)
$$

The following result holds (see also the analysis of [24, Sect.2] in the case $k=0)$.

Proposition 3.2 Let $\boldsymbol{v}_{k+1} \in \mathbb{R} \mathbb{T}_{k+1}(K)$ be uniquely decomposed as

$$
\boldsymbol{v}_{k+1}=\boldsymbol{v}_{k+1}^{0} \oplus \boldsymbol{v}_{k+1}^{\perp}(K) .
$$

The following structure can be identified

$$
\boldsymbol{v}_{k+1}^{0}=\boldsymbol{v}_{0}^{0} \oplus \sum_{l=0}^{k} \widetilde{\boldsymbol{v}}_{l+1}^{0}, \quad \boldsymbol{v}_{k+1}^{\perp}=\boldsymbol{v}_{0}^{\perp} \oplus \sum_{l=0}^{k} \widetilde{\boldsymbol{v}}_{l+1}^{\perp},
$$

where $\widetilde{\boldsymbol{v}}_{l+1} \in \widetilde{\mathbb{R}}_{l+1}(K)$ is uniquely decomposed as

$$
\widetilde{\boldsymbol{v}}_{l+1}=\widetilde{\boldsymbol{v}}_{l+1}^{0} \oplus \widetilde{\boldsymbol{v}}_{l+1}^{\perp}
$$


Proof. Set $k=0$ and let $\boldsymbol{v}_{1}^{0} \in \mathbb{R} \mathbb{T}_{1}^{0}(K)$. Setting $X=\mathbb{R} \mathbb{T}_{1}^{0}(K)$, relation (14) yields

$$
\boldsymbol{v}_{1}^{0}=\boldsymbol{v}_{0}^{0} \oplus \widetilde{\boldsymbol{v}}_{1}^{0}
$$

where $\boldsymbol{v}_{0}^{0} \in \mathbb{R} \mathbb{T}_{0}^{0}(K)$ and $\widetilde{\boldsymbol{v}}_{1}^{0} \in \widetilde{\mathbb{R}}_{1}^{0}(K)$. Set now $k=1$ and let $\boldsymbol{v}_{1}^{0} \in \mathbb{R T}_{2}^{0}(K)$. Applying again (14), yields

$$
\boldsymbol{v}_{2}^{0}=\boldsymbol{v}_{1}^{0} \oplus \widetilde{\boldsymbol{v}}_{2}^{0}=\boldsymbol{v}_{0}^{0} \oplus \sum_{l=0}^{1} \widetilde{\boldsymbol{v}}_{l+1}^{0},
$$

where $\boldsymbol{v}_{0}^{0} \in \mathbb{R T}_{0}^{0}(K)$ and $\widetilde{\boldsymbol{v}}_{l+1}^{0} \in \widetilde{\mathbb{R}}_{l+1}^{0}(K)$. Induction on $k$ gives $(25)_{1}$. Set again $k=0$ and let $\boldsymbol{v} \in \mathbb{R T}_{1}(K)$. Using (14) and then (23), yields

$$
\begin{aligned}
\boldsymbol{v}_{1} & =\boldsymbol{v}_{0} \oplus \widetilde{\boldsymbol{v}}_{1}=\left(\boldsymbol{v}_{0}^{0} \oplus \boldsymbol{v}_{0}^{\perp}\right) \oplus\left(\widetilde{\boldsymbol{v}}_{1}^{0} \oplus \widetilde{\boldsymbol{v}}_{1}^{\perp}\right) \\
& =\left(\boldsymbol{v}_{0}^{0} \oplus \widetilde{\boldsymbol{v}}_{1}^{0}\right) \oplus\left(\boldsymbol{v}_{0}^{\perp} \oplus \widetilde{\boldsymbol{v}}_{1}^{\perp}\right)
\end{aligned}
$$

Since, from $(25)_{1}$ (with $k=0$ ) we have that the term $\left(\boldsymbol{v}_{0}^{0} \oplus \widetilde{\boldsymbol{v}}_{1}^{0}\right)$ belongs to $\mathbb{R T}_{1}^{0}(K)$, this leads to recognize the following decomposition

$$
\boldsymbol{v}_{1}^{\perp}=\widetilde{\boldsymbol{v}}_{0}^{\perp} \oplus \widetilde{\boldsymbol{v}}_{1}^{\perp}
$$

Induction on $k$ yields relation $(25)_{2}$.

Proposition 3.2 has the practically relevant consequence that the hierarchical construction discussed in the previous section can be exploited also to obtain the solenoidal subspace of degree $k+1$ and its complement as completion of the corresponding subspaces of degree $k$. With this purpose, we start determining the dimensions of $\widetilde{\mathbb{R T}}_{k+1}^{0}(K)$ and $\widetilde{\mathbb{R T}}_{k+1}^{\perp}(K)$. From $(21)$, we have

$$
\operatorname{dim}\left(\widetilde{\mathbb{R T}}_{k+1}^{0}(K)\right)=\operatorname{dim}\left(\mathbb{P}_{k+2}(K)\right)-\operatorname{dim}\left(\mathbb{P}_{k+1}(K)\right)=k+3
$$

and

$$
\begin{aligned}
\operatorname{dim}\left(\widetilde{\mathbb{R T}}_{k+1}^{\perp}(K)\right) & =\left(\operatorname{dim}\left(\mathbb{R} \mathbb{T}_{k+1}(K)\right)-\operatorname{dim}\left(\mathbb{R} \mathbb{T}_{k}(K)\right)\right)-\operatorname{dim}\left(\widetilde{\mathbb{R T}}_{k+1}^{0}(K)\right) \\
& =(2 k+5)-(k+3)=k+2 .
\end{aligned}
$$

Tab. 2 summarizes the dimensions of the subspaces involved in the Helmholtz decomposition of the Raviart-Thomas finite element space of degree $k$. Notice that the table can be accessed in two different ways. Reading the table in a rwo-wise fashion informs about the dimension of the solenoidal subspace and of its complement for a given degree $k\left(e . g ., \operatorname{dim} \mathbb{R}_{2}(K)=15=\operatorname{dim} \mathbb{R} \mathbb{T}_{2}^{0}(K)+\right.$ $\left.\mathbb{R T}_{2}^{\perp}(K)=9+6\right)$. Reading the table in a "zig-zag" fashion informs about the dimension of the solenoidal subspace and of its complement viewed as the 


\begin{tabular}{|c|c|c|c|c|c|c|c|}
\hline \multirow[b]{2}{*}{$k$} & \multirow[b]{2}{*}{ space } & \multirow{2}{*}{\multicolumn{2}{|c|}{ completion }} & \multicolumn{2}{|c|}{ Solenoidal subspace } & \multicolumn{2}{|c|}{ Non-Solenoidal subspace } \\
\hline & & & & & completion & & completion \\
\hline- & $\mathbb{R} \mathbb{T}_{0}$ & 3 & - & 2 & - & 1 & - \\
\hline 0 & $\mathbb{R T}_{1}$ & 8 & 5 & 5 & 3 & 3 & 2 \\
\hline 1 & $\mathbb{R} \mathbb{T}_{2}$ & 15 & 7 & 9 & 4 & 6 & 3 \\
\hline 2 & $\mathbb{R T}_{3}$ & 24 & 9 & 14 & 5 & 10 & 4 \\
\hline 3 & $\mathbb{R} \mathbb{T}_{4}$ & 35 & 11 & 20 & 6 & 15 & 5 \\
\hline 4 & $\mathbb{R} \mathbb{T}_{5}$ & 48 & 13 & 27 & 7 & 21 & 6 \\
\hline
\end{tabular}

Table 2

Dimensions of the subspaces involved in the Helmholtz decomposition applied to the hierarchical representation of $\mathbb{R T}_{k+1}(K), k=0, \ldots, 4$. Dimensions of the respective defect corrections spaces are reported as well. Accessing the table in a row-wise fashion informs about the dimension of the full space, of its solenoidal and non-solenoidal parts. Accessing the table in a "zig-zag" fashion informs about the dimension of the solenoidal and non-solenoidal subspaces composed by the sum of the lower degree and defect correction parts.

sum of a lower degree part and of a defect correction. (e.g., $\operatorname{dim} \mathbb{R T}_{2}^{0}(K)=$ $\left.9=\operatorname{dim} \mathbb{R} \mathbb{T}_{0}^{0}(K)+\operatorname{dim} \widetilde{\mathbb{R T}}_{1}^{0}(K)+\operatorname{dim} \widetilde{\mathbb{R T}}_{2}^{0}(K)=2+3+4\right)$

It is interesting to observe that there exists a one-to-one correspondance between the alternative degrees of freedom (20) for the representation of $\widetilde{\boldsymbol{v}} \in \widetilde{\mathbb{R T}}_{k+1}(K)$ and the Helmholtz decomposition (15) of such space [13, Theorem 3.2], given by

$$
\widetilde{\boldsymbol{v}}=\nabla \widetilde{\psi}+\operatorname{curl} \tilde{\phi}, \quad \widetilde{\psi}, \tilde{\phi} \in \widetilde{\mathbb{P}}_{k+2}(K) .
$$

Precisely, denoting by $L^{A}(\boldsymbol{v}), L^{B}(\boldsymbol{v})$ and $L^{C}(\boldsymbol{v})$ the linear functionals expressing the degrees of freedom in $\Sigma_{A}, \Sigma_{B}$ and $\Sigma_{C}$, respectively, we have

$$
\begin{aligned}
\nabla \widetilde{\psi} & =\sum_{i=1}^{k+2} L_{i}^{B}(\boldsymbol{v}) \boldsymbol{\tau}_{i}^{B}, \\
\operatorname{curl} \widetilde{\phi} & =\sum_{i=1}^{3} L_{i}^{A}(\boldsymbol{v}) \boldsymbol{\tau}_{i}^{A}+\sum_{i=1}^{k} L_{i}^{C}(\boldsymbol{v}) \boldsymbol{\tau}_{i}^{C}
\end{aligned}
$$

$\boldsymbol{\tau}_{i}^{A}, \boldsymbol{\tau}_{i}^{B}$ and $\boldsymbol{\tau}_{i}^{C}$ being the basis functions related to $\Sigma_{A}, \Sigma_{B}$ and $\Sigma_{C}$.

The degrees of freedom of the hierarchical-Helmholtz decomposition are depicted in Fig. 1 in the cases $k=0,1,2,3$. Notice that in the special case $k=0$, the hierarchical strategy does not apply, while an Helmholtz decomposition 


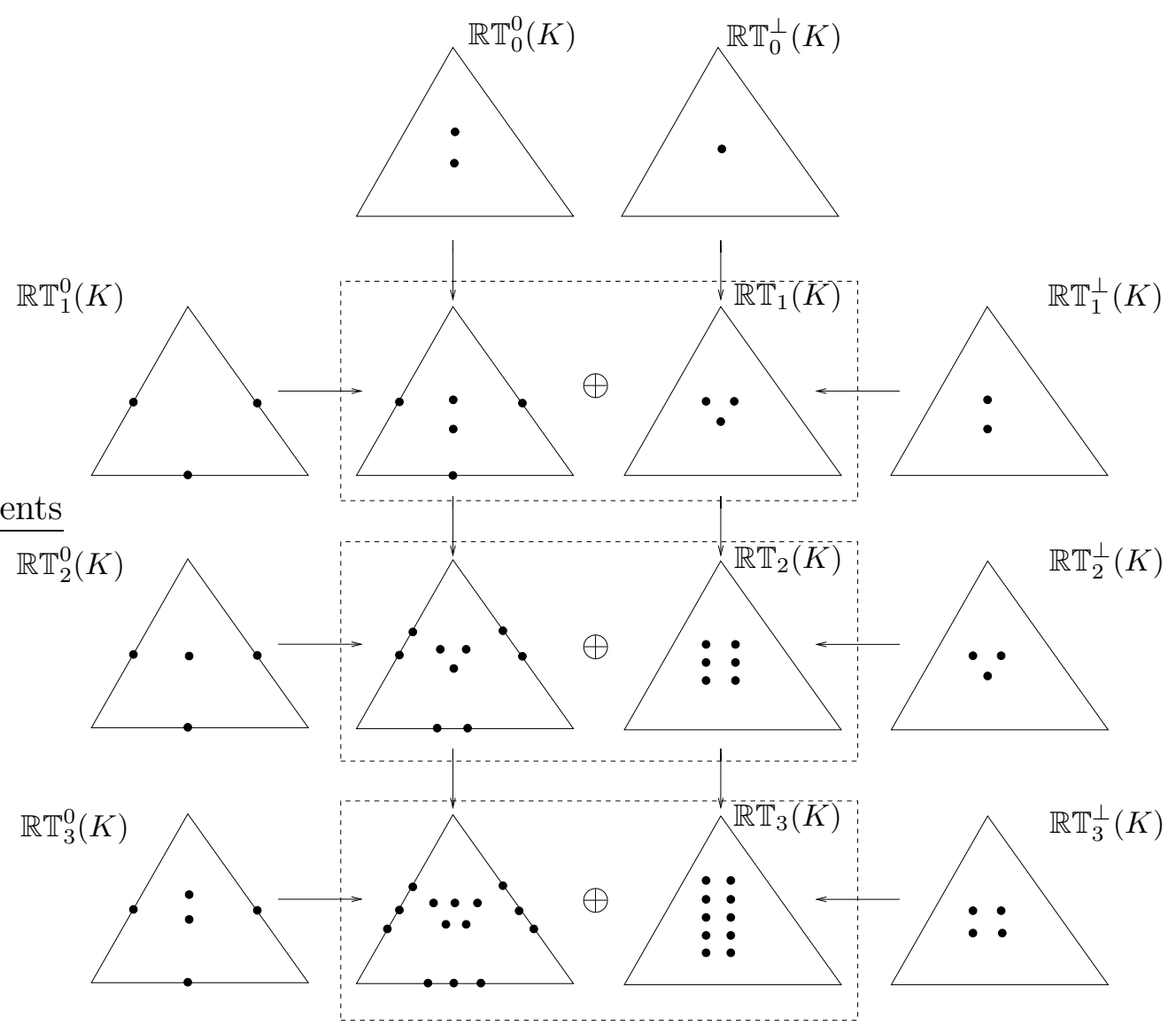

Figure 1. Construction of the space $\mathbb{R T}_{k}(K)$ for $k=1,2,3$. In the leftmost column, the solenoidal defect correction degrees of freedom are shown, in the rightmost column the non-solenoidal defect correction degrees of freedom are shown. Contributions from the lower degree subspace are represented by the vertical arrows. The two central columns show the solenoidal (left) and non-solenoidal (right) subspaces of the $\mathbb{R T}_{k}(K)$ space.

can be carried out (cf. Sect.3.3 for details).

\subsection{An Example of Computation of Basis Functions}

In this section, we carry out the computation of the basis functions for the finite element spaces $\mathbb{R}_{1}(K) \times \mathbb{P}_{1}(K) \times R_{1}(\partial K)$. With this aim, it is useful to refer to the half unit-square triangle $\widehat{K}$ (reference element), this approach being the most efficient and flexible for coding the method on the computer. Let 
$\widehat{\mathbf{x}}=(\widehat{x}, \widehat{y})^{t}$ be the coordinate vector in $\widehat{K}$. We define the affine transformation

$$
\begin{aligned}
& F_{K}: \widehat{K} \rightarrow K, \\
& F_{K}(\widehat{\boldsymbol{x}})=\mathbf{b}_{K}+B_{K} \widehat{\boldsymbol{x}}, \quad \text { with } \quad \mathbf{b}_{K}=\left[\begin{array}{l}
x_{1} \\
y_{1}
\end{array}\right], B_{K}=\left[\begin{array}{ll}
x_{2}-x_{1} & x_{3}-x_{1} \\
y_{2}-y_{1} & y_{3}-y_{1}
\end{array}\right]
\end{aligned}
$$

which maps the reference triangle into the element $K \in \mathcal{T}_{h}$ of vertices $\left(x_{i}, y_{i}\right), i=$ $1,2,3$, labelled with a counterclockwise orientation. Accordingly, for a scalarvalued function $w$ and a vector-valued function $\tau$ defined on $K$, we set

$$
w(\boldsymbol{x})=\widehat{w}(\widehat{\boldsymbol{x}}), \quad \boldsymbol{\tau}(\boldsymbol{x})=\mathfrak{P}_{K} \widehat{\boldsymbol{\tau}}(\widehat{\boldsymbol{x}}),
$$

where $\mathfrak{P}_{K}$ is the Piola transformation (cf. [4], Sect. III.1.3) such that

$$
\begin{aligned}
& \mathfrak{P}_{K}:\left(L^{2}(\widehat{K})\right)^{2} \rightarrow\left(L^{2}(K)\right)^{2}, \\
& \mathfrak{P}_{K} \widehat{\boldsymbol{\tau}}(\widehat{\boldsymbol{x}})=\frac{1}{m_{K}} B_{K} \widehat{\boldsymbol{\tau}}(\widehat{\boldsymbol{x}}), \text { with } \quad m_{K}:=\left|\operatorname{det} B_{K}\right|
\end{aligned}
$$

Enforcing $L^{2}$ orthogonality on the $1 \mathrm{D}$ reference element $\widehat{e}=(-1,1)$, we get $\mathbb{P}_{0}(\widehat{e})=\operatorname{span}\{1\}$ and $\widetilde{\mathbb{P}}_{1}(\widehat{e})=\operatorname{span}\{s\}, s$ being the curvilinear abscissa on $\widehat{e}$. Enforcing $L^{2}$ orthogonality on $\widehat{K}$, we get $\mathbb{P}_{0}(\widehat{K})=\operatorname{span}\{1\}$, and $\widetilde{\mathbb{P}}_{1}(\widehat{K})=$ $\operatorname{span}\{\widehat{x}-1 / 3, \widehat{y}-1 / 3\}$. We come now to the vector-valued finite element space. The standard basis functions associated with the lower degree space $\mathbb{R} \mathbb{T}_{0}(\widehat{K})$ are

$$
\widehat{\boldsymbol{\tau}}_{1}=\left[\begin{array}{l}
\widehat{x} \\
\widehat{y}
\end{array}\right], \quad \widehat{\boldsymbol{\tau}}_{2}=\left[\begin{array}{c}
\widehat{x} \\
\widehat{y}-1
\end{array}\right], \quad \widehat{\boldsymbol{\tau}}_{3}=\left[\begin{array}{c}
\widehat{x}-1 \\
\widehat{y}
\end{array}\right]
$$

The basis functions of the defect correction space can be constructed from the degrees of freedom (20). Namely, the basis functions associated with the degrees of freedom of Type-A (edge modes) are

$$
\widehat{\boldsymbol{\tau}}_{4}=\left[\begin{array}{c}
-3 \sqrt{2} \widehat{x} \\
3 \sqrt{2} \widehat{y}
\end{array}\right], \quad \widehat{\boldsymbol{\tau}}_{5}=\left[\begin{array}{c}
-6+6 \widehat{x}+12 \widehat{y} \\
-6 \widehat{y}
\end{array}\right], \quad \widehat{\boldsymbol{\tau}}_{6}=\left[\begin{array}{c}
6 \widehat{x} \\
6-12 \widehat{x}-6 \widehat{y}
\end{array}\right],
$$

while the basis functions associated with the degrees of freedom of Type-B (internal modes) are

$$
\widehat{\boldsymbol{\tau}}_{7}=\left[\begin{array}{c}
-2 \widehat{x}+2 \widehat{x}^{2}+\widehat{x} \widehat{y} \\
-\widehat{y}+2 \widehat{x} \widehat{y}+\widehat{y}^{2}
\end{array}\right], \quad \widehat{\boldsymbol{\tau}}_{8}=\left[\begin{array}{c}
-\widehat{x}+\widehat{x}^{2}+2 \widehat{x} \widehat{y} \\
-2 \widehat{y}+\widehat{x} \widehat{y}+2 \widehat{y}^{2}
\end{array}\right] .
$$


In the present case, $k=0$, there are no degrees of freedom of Type-C. Summarizing, we have that $\mathbb{R T}_{0}(\widehat{K})=\operatorname{span}\left\{\widehat{\boldsymbol{\tau}}_{1}, \widehat{\boldsymbol{\tau}}_{2}, \widehat{\boldsymbol{\tau}}_{3}\right\}, \widetilde{\mathbb{R T}}_{1}^{0}(\widehat{K})=\operatorname{span}\left\{\widehat{\boldsymbol{\tau}}_{4}, \widehat{\boldsymbol{\tau}}_{5}, \widehat{\boldsymbol{\tau}}_{6}\right\}$ and $\widetilde{\mathbb{R T}}_{1}^{\perp}(\widehat{K})=\operatorname{span}\left\{\widehat{\boldsymbol{\tau}}_{7}, \widehat{\boldsymbol{\tau}}_{8}\right\}$. Fig. 2 depicts the basis functions $\widehat{\tau}_{1}, \ldots, \widehat{\tau}_{8}$.

Remark 3.1 For each $e \in \partial \widehat{K}$, the following properties hold

$$
\begin{array}{lll}
\text { i) } & \int_{e} \widehat{\boldsymbol{\tau}}_{i} \cdot \widehat{\boldsymbol{n}} d \widehat{s}=0, & i=4,5,6, \\
\text { ii) } & \left.\widehat{\boldsymbol{\tau}}_{i} \cdot \widehat{\boldsymbol{n}}\right|_{e}=0, \quad \int_{\widehat{K}} \operatorname{div} \widehat{\boldsymbol{\tau}}_{i} d \widehat{x}=0, & i=7,8 .
\end{array}
$$

We notice that the basis functions $\widehat{\tau}_{4}, \ldots, \widehat{\tau}_{8}$ were computed also in [1], producing a different result which does not satisfy properties i) and ii). Moreover, if we use the present basis functions to compute on the reference triangle the constant $\gamma_{1 K}$ of the Strengthened Cauchy-Buniakowskii-Schwarz (CBS) inequality as in [1, Sect.3.2], we obtain the value $\gamma_{1 K}=0.8185$ (to be compared with the value 0.9486 found in [1]).

Remark 3.2 Applying the Helmholtz decomposition also to the space $\mathbb{R T}_{0}(\widehat{K})$ yields

$$
\widehat{\boldsymbol{\tau}}_{1}=\left[\begin{array}{l}
1 \\
0
\end{array}\right], \quad \widehat{\boldsymbol{\tau}}_{2}=\left[\begin{array}{l}
0 \\
1
\end{array}\right], \quad \widehat{\boldsymbol{\tau}}_{3}=\left[\begin{array}{c}
\widehat{x}-1 / 3 \\
\widehat{y}-1 / 3
\end{array}\right],
$$

from which we have the alternative representation

$$
\mathbb{R} \mathbb{T}_{0}^{0}(\widehat{K})=\operatorname{span}\left\{\widehat{\boldsymbol{\tau}}_{1}, \widehat{\boldsymbol{\tau}}_{2}\right\}, \quad \mathbb{R T}_{0}^{\perp}(\widehat{K})=\operatorname{span}\left\{\widehat{\boldsymbol{\tau}}_{3}\right\}
$$

\section{Decoupled DMH Problem with Approximate Flux Mass Matrix}

In this section, we discuss the structure of problem (9) when the basis functions introduced in Sect. 3 are used and we propose a computational procedure for the effective solution of the resulting system. We assume in the following that $\sigma$ is a piecewise constant nonnegative function on $\mathcal{T}_{h}$.

Given a generic operator $\mathcal{X}$, we denote by $\mathcal{X}^{K}$ its restriction to each $K \in \mathcal{T}_{h}$. We associate with the index 1 the finite element spaces of lower degree in the hierarchical decomposition (for both scalar and vector-valued functions), while the index 2 denotes the defect correction space (for scalar-valued functions) or its divergence-free part (for vector-valued functions) and the index 3 denotes the remaining part of the defect correction space (for vector-valued functions). Then, with the notation $\mathcal{X}_{\mu, \nu}^{K}, \mu, \nu=1,2,3$, we denote the operator acting between the space labelled with $\mu$ and the dual of the space labelled with $\nu$. 

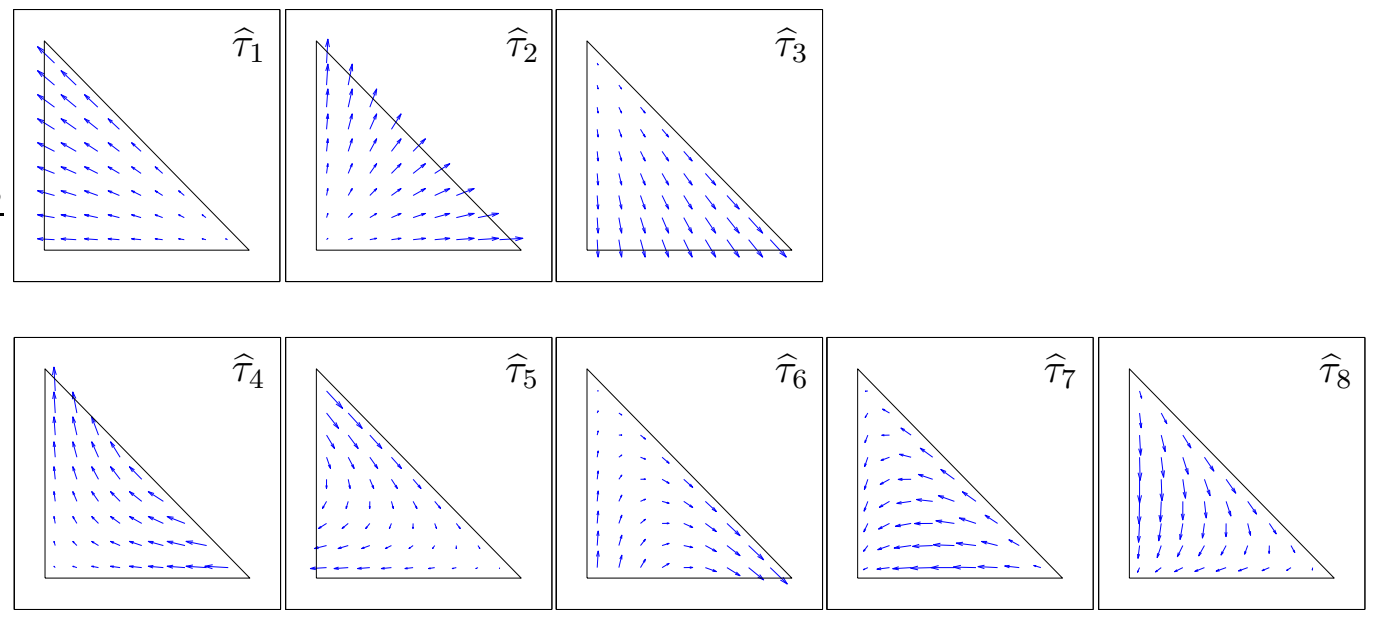

Figure 2. Basis functions $\widehat{\tau}_{1}, \ldots, \widehat{\tau}_{8}$. Functions in the first row span the space $\mathbb{R T}_{0}(\widehat{K})$, functions in the second row span the defect correction space $\widetilde{R T}_{1}(\widehat{K})$. Functions $\widehat{\tau}_{4}, \widehat{\tau}_{5}, \widehat{\tau}_{6}$ are solenoidal edge modes, functions $\widehat{\tau}_{7}, \widehat{\tau}_{8}$ are non-solenoidal internal modes. Notice that $\widehat{\tau}_{4}, \widehat{\tau}_{5}, \widehat{\tau}_{6}$ have null flux on each edge of $\partial \widehat{K}$, while $\widehat{\tau}_{7}, \widehat{\tau}_{8}$ have null normal component on each edge of $\partial \widehat{K}$.

Let us consider problem (9) written -with a simple shift of indices- for the degree $k+1, k \geq 0$, and let us assume that each set of variables is represented using the decompositions (14)-(15) and is labelled according to the above mentioned convention. Due to the properties of the hierarchical-Helmholtz decomposition, we have that, if $\mu \neq \nu$,

$$
\begin{aligned}
& \left(\mathcal{B}_{\mu, \nu}^{K} \boldsymbol{v}_{\nu}, w_{\mu}\right)_{\mathbb{P}_{\mu}(K)}=0 \\
& \left(\mathcal{C}_{\mu, \nu}^{K} \boldsymbol{v}_{\nu}, \eta_{\mu}\right)_{R_{\mu}(\partial K)}=0 \\
& \left(\mathcal{D}_{\mu, \nu}^{K} u_{\nu}, w_{\mu}\right)_{\mathbb{P}_{\mu}(K)}=0 .
\end{aligned}
$$

Moreover,

$$
\begin{array}{ll}
\left(\mathcal{B}_{\mu, \nu}^{K} \boldsymbol{v}_{\nu}, w_{\mu}\right)_{\mathbb{P}_{\mu}(K)}=0 & \text { if } \nu=2, \\
\left(\mathcal{C}_{\mu, \nu}^{K} \boldsymbol{v}_{\nu}, \eta_{\mu}\right)_{R_{\mu}(\partial K)}=0 & \text { if } \nu=3 .
\end{array}
$$

Relations (29) imply a complete separation between subspaces in the bilinear forms $b, c$ and $d$. Relation $(30)_{1}$ implies that the divergence-free part of the defect correction space cannot contribute to lift any given load $f$, while relation $(30)_{2}$ implies a complete localization of problems arising from the non-solenoidal part of the defect correction subspace. As a matter of fact, coupling occurs because the spaces $\mathbb{R T}_{k}(K), \widetilde{\mathbb{R T}}_{k+1}^{0}(K)$ and $\widetilde{\mathbb{R T}}_{k+1}^{\perp}(K)$ are not orthogonal with respect to the bilinear form $a$, so that some form of diagonalization may be envisaged to reduce the computational effort, ending up with an approximate flux mass matrix. In order to study the effect of operating a diagonalization of $\mathcal{A}^{K}$, we analyze more in detail its structure. We 
have

$$
\left(\mathcal{A}^{K} \boldsymbol{q}_{k+1}, \boldsymbol{v}_{k+1}\right)_{\boldsymbol{V}_{k+1}\left(\Omega ; \mathcal{T}_{h}\right)}=\left(\left(\begin{array}{ccc}
\mathcal{A}_{1,1} & \mathcal{A}_{1,2} & \mathcal{A}_{1,3} \\
\mathcal{A}_{1,2}^{t} & \mathcal{A}_{2,2} & \mathcal{A}_{2,3} \\
\mathcal{A}_{1,3}^{t} & \mathcal{A}_{2,3}^{t} & \mathcal{A}_{3,3}
\end{array}\right)\left(\begin{array}{c}
\boldsymbol{q}_{k} \\
\widetilde{\boldsymbol{q}}_{k+1}^{0} \\
\widetilde{\boldsymbol{q}}_{k+1}^{\perp}
\end{array}\right),\left(\begin{array}{c}
\boldsymbol{v}_{k} \\
\widetilde{\boldsymbol{v}}_{k+1}^{0} \\
\widetilde{\boldsymbol{v}}_{k+1}^{\perp}
\end{array}\right)\right)
$$

where $\mathcal{A}$ acts on $\boldsymbol{q}$ in the standard form of a matrix-vector product. Care must be paid to ensure that the modified operator preserves the theoretical convergence order of the DMH method of degree $k+1$ [4]. This issue is the subject of the forthcoming section.

\subsection{Error Analysis for the Approximate DMH Formulation: Numerical Val- idation}

In the following, we consider two numerical examples for which the exact solution is available and we monitor the convergence rates obtained for different choices of the diagonalization procedure in the case of RT elements of degree 1. In both examples, $\Omega=(0,1)^{2}, g_{D}=0$ and $f$ is such that the exact solution is the function $u=16 x y(x-1)(y-1)$.

- Test 1: we solve problem (1) setting $\kappa=1$ and $\sigma=1$.

- Test 2: we solve problem (1) setting $\kappa=1+\alpha \mathrm{e}^{-\beta\left((x-1 / 2)^{2}+(y-1 / 2)^{2}\right)}$, with $\alpha=1, \beta=10$, and $\sigma=0$.

Notice that, despite the fact that Test 1 and Test 2 have the same exact solution $u$, in Test $1, \kappa$ and $\sigma$ are constant functions, but the non-null reaction term strongly couples the primal and the dual variable. In Test $2, \kappa$ is instead a function of the position, and this introduces a strong coupling in the flux mass matrix. In Figs. 3 and. 4 we show the convergence rates corresponding to setting to zero the extra-diagonal blocks of $\mathcal{A}_{\mu, \nu}^{K}, \mu \neq \nu$, as specified in the respective legends, using right-angled meshes with size $h=[1 / 2,1 / 5,1 / 20,1 / 40,1 / 80]$. Given the corresponding discrete solutions $\left(\boldsymbol{q}_{h}, u_{h}, \lambda_{h}\right)$, we plot the quantities $\left\|\boldsymbol{q}-\boldsymbol{q}_{h}\right\|_{\text {div; } \Omega},\left\|u-u_{h}\right\|_{0 ; \Omega}$ and $\left\|\mathcal{P} u-\lambda_{h}\right\|_{-1 / 2, h}$, this latter norm being defined as $[4]$

$$
\|\eta\|_{-1 / 2, h}:=\left(\sum_{e \in \mathcal{E}_{h}} \mid e\|\| \eta \|_{0 ; e}^{2}\right)^{1 / 2}
$$

where $\mathcal{P}$ is the $L^{2}$ projection onto $\mathbb{P}_{k}(e), e \in \mathcal{E}_{h}$. The same legend is used in all figures: $\mathbb{R T}_{0}$ (red): 'o', $\mathbb{R} \mathbb{T}_{1}$ (blue): ' $\square$ ', $\mathcal{A}_{12}, \mathcal{A}_{13}, \mathcal{A}_{23}=0$ (green): ' $\nabla$ ', $\mathcal{A}_{23}=0$ (magenta): '×', $\mathcal{A}_{12}, \mathcal{A}_{23}=0$ (cyan): ' $\triangleleft$ ', $\mathcal{A}_{13}, \mathcal{A}_{23}=0$ (dark yellow): ' $\triangleright$ '.

Both sets of computations indicate that the optimal rate of convergence of the plain DMH method using RT elements of degree 1 is retained only in the 



Figure 3. Convergence rates for Test 1 obtained for different choices of the approxi-


$\left\|\mathcal{P} u-\lambda_{h}\right\|_{-1 / 2, h}$. The same legend is used in all figures: $\mathbb{R T}_{0}$ (red): 'o', $\mathbb{R T}_{1}$ (blue): ' $\square$ ', $\mathcal{A}_{12}, \mathcal{A}_{13}, \mathcal{A}_{23}=0$ (green): ' $\nabla$ ', $\mathcal{A}_{23}=0$ (magenta): ' ' ', $\mathcal{A}_{12}, \mathcal{A}_{23}=0$ (cyan): ' $\triangleleft$ ', $\mathcal{A}_{13}, \mathcal{A}_{23}=0$ (dark yellow): ' $\triangleright$ '.

case where the sole $\mathcal{A}_{23}$ coupling term is neglected. This is supported by the fact that in all figures the error curves associated with the choice $\mathcal{A}_{23}=0$ are superposed or parallel to the corresponding error curve in the case of the plain DMH method. We also notice that a complete block diagonalization $\mathcal{A}_{12}, \mathcal{A}_{13}, \mathcal{A}_{23}=0$ spoils the accuracy of the method with particular effect on the convergence of the hybrid variable. This latter effect is a direct consequence of the lack of accuracy in the approximation of the internal variables (cf. the error estimates for the hybrid variable proved in [2] in the case of Galerkin formulations and in [7] in the case of Petrov-Galerkin formulations). However, the full block diagonalization of $\mathcal{A}$ can still be profitably used in the context of a-posteriori error estimation, because in this application the solution obtained from the approximation of degree 1 is sufficient to produce a consistent error estimator for the solution of degree 0 (see [24] for a discussion of this topic and [17] for the generalization to an approximation of arbitrary degree).

We conclude this section by pointing out that an extensive numerical validation, carried out on several other test problems also employing unstructured 

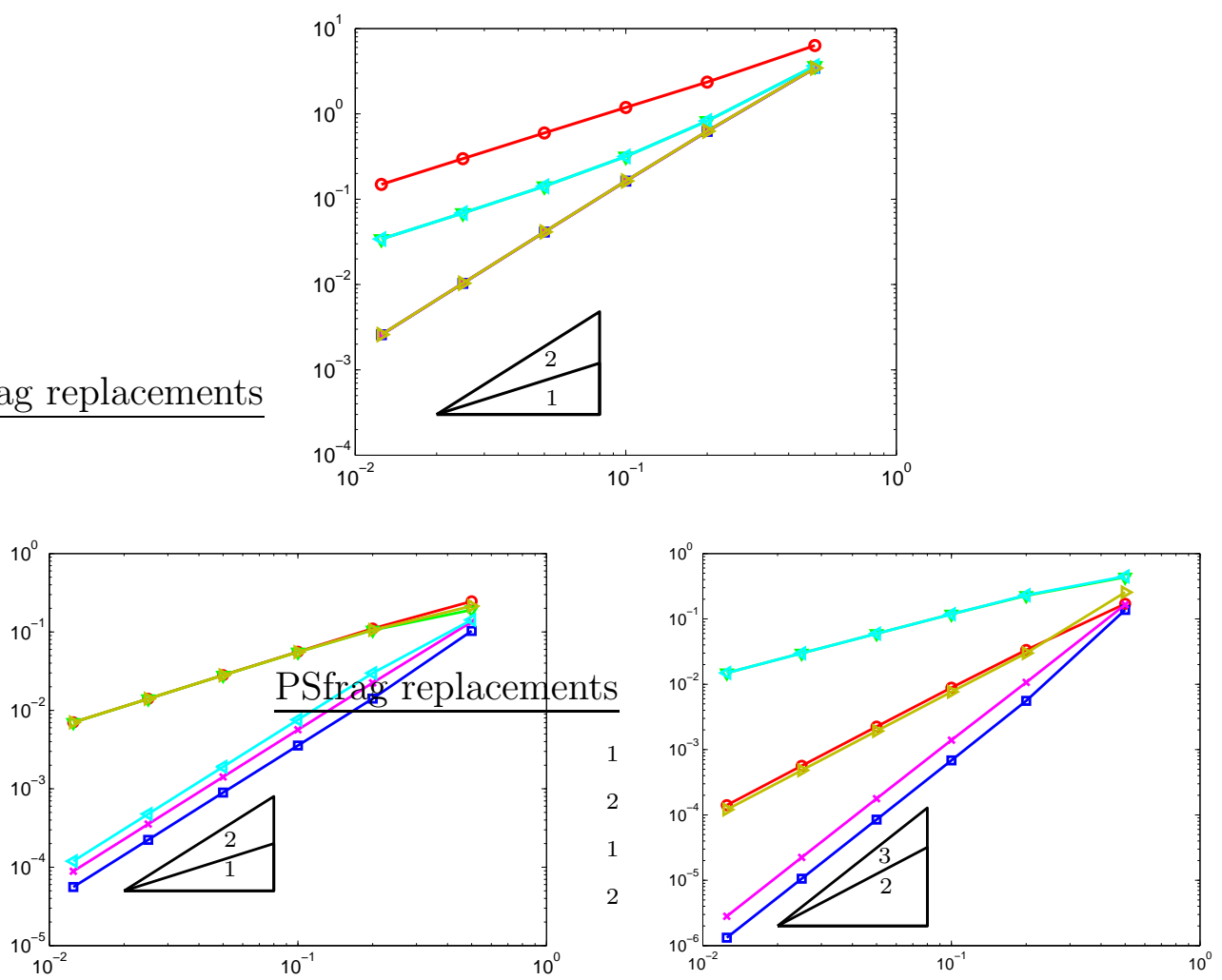

Figure 4. Convergence rates for Test 2 obtained for different choices of the approximate flux mass matrix. Top: || $\boldsymbol{q}-\boldsymbol{q}_{h} \mid \|_{\text {div; } \Omega}$. Bottom left: $\left\|u-u_{h}\right\|_{0 ; \Omega}$, bottom right: $\left\|\mathcal{P} u-\lambda_{h}\right\|_{-1 / 2, h}$. The same legend is used in all figures: $\mathbb{R T}_{0}$ (red): 'o', $\mathbb{R T}_{1}$ (blue): ' $\square$ ', $\mathcal{A}_{12}, \mathcal{A}_{13}, \mathcal{A}_{23}=0$ (green): ' $\nabla$ ', $\mathcal{A}_{23}=0$ (magenta): ' ' ', $\mathcal{A}_{12}, \mathcal{A}_{23}=0$ (cyan): ' $\triangleleft$ ', $\mathcal{A}_{13}, \mathcal{A}_{23}=0$ (dark yellow): ' $\triangleright$ '.

grids, yields completely analogous results.

\subsection{Fixed-Point Iteration}

The general trend suggested by the discussion in Sect. 4.1 indicates that a complete decoupling between different scales in the hierarchical approximation spoils the accuracy of the method. In order to take advantage of the hierarchical structure without a degradation of the optimal rate of convergence, we consider a fixed-point iteration based on the successive choice in (9) of the sets of test functions $\left(\boldsymbol{v}_{k}, w_{k}, \eta_{k}\right),\left(\widetilde{\boldsymbol{v}}_{k+1}^{0}, \widetilde{w}_{k+1}, 0\right)$ and $\left(\widetilde{\boldsymbol{v}}_{k+1}^{\perp}, 0, \widetilde{\eta}_{k+1}\right)$.

Given the initial guess solution $\left(\boldsymbol{q}_{k+1}, u_{k+1}, \lambda_{k+1}\right)^{(0)}$ and an input tolerance tol, the iterative procedure consists of the following steps, for $\ell \geq 0$ : 
- Step S1. Solve the lower degree problem:

$$
\left(\begin{array}{ccc}
\mathcal{A}_{1,1} & \mathcal{B}_{1,1}^{t} & \mathcal{C}_{1,1}^{t} \\
\mathcal{B}_{1,1} & \mathcal{D}_{1,1} & 0 \\
\mathcal{C}_{1,1} & 0 & 0
\end{array}\right)\left(\begin{array}{l}
\boldsymbol{q}_{k} \\
u_{k} \\
\lambda_{k}
\end{array}\right)^{(\ell+1)}=\left(\begin{array}{c}
-\mathcal{A}_{1,2} \widetilde{\boldsymbol{q}}_{k+1}^{0}-\mathcal{A}_{1,3} \widetilde{\boldsymbol{q}}_{k+1}^{\perp} \\
0 \\
0
\end{array}\right)^{(\ell)}+\left(\begin{array}{c}
\mathcal{G}_{1} \\
\mathcal{F}_{1} \\
0
\end{array}\right)
$$

- Step S2. Solve the solenoidal defect correction problem:

$$
\left(\begin{array}{cc}
\mathcal{A}_{2,2} & \mathcal{C}_{2,2}^{t} \\
\mathcal{C}_{2,2} & 0
\end{array}\right)\left(\begin{array}{l}
\widetilde{\boldsymbol{q}}_{k+1}^{0} \\
\widetilde{\lambda}_{k+1}
\end{array}\right)^{(\ell+1)}=\left(\begin{array}{c}
-\mathcal{A}_{1,2}^{t} \boldsymbol{q}_{k} \\
0
\end{array}\right)^{(*)}+\left(\begin{array}{c}
\mathcal{G}_{2} \\
0
\end{array}\right)
$$

- Step S3. Solve on each $K \in \mathcal{T}_{h}$ the non-solenoidal defect correction problem:

$$
\left(\begin{array}{cc}
\mathcal{A}_{3,3}^{K} & \left(\mathcal{B}_{3,3}^{K}\right)^{t} \\
\mathcal{B}_{3,3}^{K} & \mathcal{D}_{2,2}^{K}
\end{array}\right)\left(\begin{array}{l}
\widetilde{\boldsymbol{q}}_{k+1}^{\perp} \\
\widetilde{u}_{k+1}
\end{array}\right)^{(\ell+1)}=\left(\begin{array}{c}
-\left(\mathcal{A}_{1,3}^{K}\right)^{t} \boldsymbol{q}_{k} \\
0
\end{array}\right)^{(*)}+\left(\begin{array}{c}
0 \\
\mathcal{F}_{2}^{K}
\end{array}\right)
$$

- Step S4. If $\left\|\left|\boldsymbol{q}_{k+1}^{(\ell+1)}-\boldsymbol{q}_{k}^{(\ell)}\right|\right\|_{H(\text { div } ; \Omega)} \geq$ tol, then $\ell:=\ell+1$ and go to Step S1, otherwise stop.

The well-posedness of the saddle-point defect correction problems (33) and (34) can be proved by a proper adaptation of [4, Lemma V.I.2] and of [20, Lemma 7.2.1], respectively.

Notice that a block Gauss-Seidel iteration is obtained if $(\cdot)^{*}=(\cdot)^{(\ell+1)}$, while a completely parallelizable block Jacobi iteration is recovered if $(\cdot)^{*}=(\cdot)^{\ell}$. Our computational tests indicate that at most 6-7 Gauss-Seidel iterations are required to obtain an accuracy of the order of $10^{-7}$ for the increment $\left(\boldsymbol{q}_{1}^{\ell+1}-\boldsymbol{q}_{1}^{\ell}\right)$ measured in the maximum vector norm, with a decreasing trend as the mesh size goes to zero (see also the comments at the end of Sect. 6).

\subsubsection{Remarks on the Implementation}

Step S1 requires solving a saddle point problem in the lower degree variables, that can be treated by static condensation (see Sect. 5), yielding a symmetric definite positive system of the form (13) for the sole hybrid variable. Since the coefficient matrix does not depend on $\ell$, it can be factorized once for all, in such a way that a lower and a triangular system must be solved at each iteration. The same holds for Step S2. Observe that the system associated with Step S2 written in condensed form has dimension $\mathrm{Ni}$ irrespective of the approximation degree $k$, because $\mathrm{Ni}$ is the number of degrees of freedom of the defect correction space of degree $k+1$ of the Lagrange multiplier. Step 


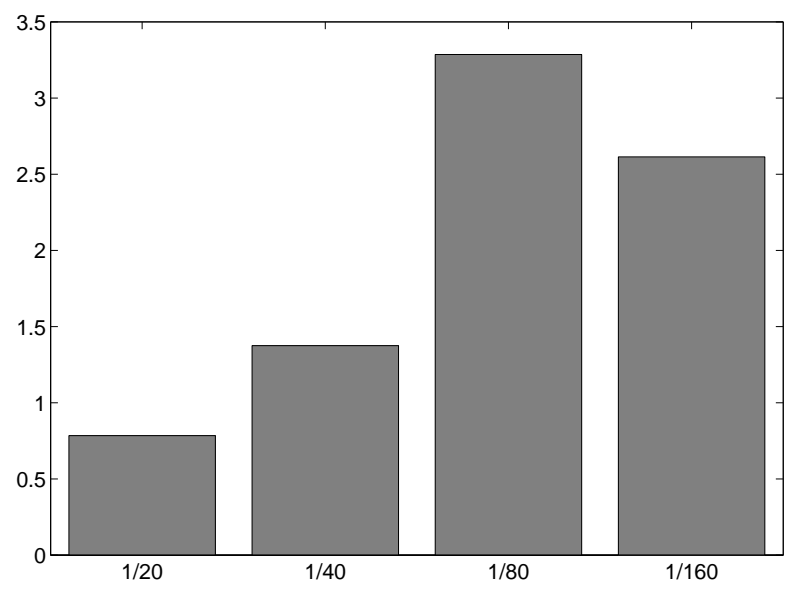

Figure 5. Ratio $T_{\text {plain }} / T_{\text {approx }}$ as a function of the mesh size $h$.

S3 requires solving Ne local saddle-point problems. If $\sigma=0$, the variable $\widetilde{\boldsymbol{q}}_{k+1}^{\perp}$ can be uniquely determined from $(34)_{2}$, the matrix associated with $\mathcal{B}_{3,3}^{K}$ being square and invertible. Such a computation can be carried out once for all at the beginning of the fixed point iteration. The quantity $\widetilde{u}_{k+1}$, that does not enter the iterations as well, can be computed, if required, at the end of the process. Observe that in Steps S2 and S3 the coupling between the defect correction spaces can be neglected, according to the observations of Sect. 4.1.

If the computer code is to be implemented in MATLAB, attention must be paid also to Step S3, that apparently is the cheapest one. As a matter of fact, looping on mesh elements for the construction of local matrices introduces a severe bottleneck, even when an appropriate and massive vectorization of operations is carried out. This step may indeed be critical with respect to the time required to solve linear systems using the efficient built-in MATLAB functions (as in Steps S1 and S2). However, since the coefficient matrices do not change from iteration to iteration in the algorithm proposed above, an advantageous strategy is to store them at the beginning of the iterative procedure, an obvious trade-off being needed, for very large meshes, between memory requirements and efficiency of computation.

In order to assess the efficiency of the proposed approximate $\mathrm{DMH}$ formulation, we compare the execution time $T_{\text {plain }}$ of the plain hybridized method and the execution time $T_{\text {approx }}$ of the present approach in the solution of Test 1 (described in Sect. 4.1) on four uniform triangulations of size $h=$ $[1 / 20,1 / 40,1 / 80,1 / 160]$. Fig 5 shows the ratio $T_{\text {plain }} / T_{\text {approx }}$ as a function of the mesh size $h$, and clearly demonstrates the substantial improvement in reducing the computational effort provided by the approximate formulation compared to the plain DMH method. 


\subsubsection{Properties of DMH approximations of different degree}

In this section, we illustrate the properties that a solution of the DMH method of degree $k+1$ inherits from a solution of degree $k, k \geq 0$. Let $\boldsymbol{q}^{[s]}=\sum_{l=0}^{s} \widetilde{\boldsymbol{q}}_{l}^{[s]}$, $u^{[s]}=\sum_{l=0}^{s} \widetilde{u}_{l}^{[s]}, \lambda^{[s]}=\sum_{l=0}^{s} \widetilde{\lambda}_{l}^{[s]}$ be the solution of the DMH problem obtained using finite element spaces of degree $s \geq 0$, with $(\widetilde{\boldsymbol{q}}, \widetilde{u}, \widetilde{\lambda})_{0}^{[s]}:=(\boldsymbol{q}, u, \lambda)_{0}^{[s]}$. Notice that superscripts are added here for clarity to highlight the fact that each solution component refers to the approximation space of degree $s$. Moreover, we set $\operatorname{osc}_{0}(f):=\Pi_{0} f$ and for $s \geq 1$, we let $\operatorname{osc}_{s}(f):=\Pi_{s} f-\Pi_{s-1} f$, where $\Pi_{k}$ is the $L^{2}$ projection onto $\mathbb{P}_{k}(K)$. Then, the following properties hold:

- Property P1 (Self-equilibrium):

$$
\operatorname{div} \boldsymbol{q}_{k}^{[k]}(\boldsymbol{x})=\Pi_{k} f(\boldsymbol{x})-\left.\sigma\right|_{K} u_{k}(\boldsymbol{x}), \quad \boldsymbol{x} \in K .
$$

Moerover, if $\left.\sigma\right|_{K}=0$

$$
\begin{aligned}
& \operatorname{div} \widetilde{\boldsymbol{q}}_{l}^{[k+1]}(\boldsymbol{x})=\operatorname{div} \widetilde{\boldsymbol{q}}_{l}^{[k]}(\boldsymbol{x})=\operatorname{osc}_{l}(f)(\boldsymbol{x}), \quad l=0, \ldots, k, \\
& \operatorname{div} \widetilde{\boldsymbol{q}}_{k+1}^{[k+1]}(\boldsymbol{x})=\operatorname{osc}_{k}(f)(\boldsymbol{x}) .
\end{aligned}
$$

- Property P2 (Flux conservation):

$$
\int_{\partial K}\left(\boldsymbol{q}^{[k+1]}-\boldsymbol{q}^{[k]}\right) \cdot \boldsymbol{n} d s=\int_{\partial K}\left(\boldsymbol{q}_{0}^{[k+1]}-\boldsymbol{q}_{0}^{[k]}\right) \cdot \boldsymbol{n} d s=\sigma_{K} \int_{K}\left(u_{0}^{[k+1]}-u_{0}^{[k]}\right) d x
$$

- Property P3 (Interelement reciprocity of normal fluxes):

$$
\int_{e} \llbracket \widetilde{\boldsymbol{q}}_{l}^{[k+1]} \rrbracket \widetilde{\eta}_{l} d s=\int_{e} \llbracket \widetilde{\boldsymbol{q}}_{l}^{[k]} \rrbracket \widetilde{\eta}_{l} d s, \quad l=0, \ldots, k,
$$

where $\llbracket \boldsymbol{q} \rrbracket=\left.\boldsymbol{q} \cdot \boldsymbol{n}\right|_{\partial K_{1}}+\left.\boldsymbol{q} \cdot \boldsymbol{n}\right|_{\partial K_{2}}, e=\partial K_{1} \cap \partial K_{2}, e \in \mathcal{E}_{h, i}$, is the jump of the normal component of the flux across $e$.

\section{Solution of Approximate DMH Problems in Condensed Form}

In order to efficiently solve the systems appearing in Steps S1 and S2 of the previous section, it is useful to employ the approach of [9] for obtaining the matrix $E$ and the vector $H$ of the condensed system (13). The solution of problem in Step S1 follows the same lines as in [9] with just a minor change due to the presence of an additional right-hand side in $(32)_{1}$. As for the system in Step S2, we proceed considering the local lifting of $\widetilde{\lambda}_{k+1}$ as if this latter 
quantity were a given boundary variable on $\partial K$. Then, for all $K \in \mathcal{T}_{h}$, we seek $\widetilde{\boldsymbol{q}}_{k+1, \widetilde{\lambda}}^{0} \in \widetilde{\mathbb{R}}_{k+1}^{0}(K)$ such that

$$
a^{K}\left(\widetilde{\boldsymbol{q}}_{k+1, \widetilde{\lambda}}^{0}, \widetilde{\boldsymbol{v}}_{k+1}^{0}\right)=-\int_{\partial K} \widetilde{\lambda}_{k+1} \widetilde{\boldsymbol{v}}_{k+1}^{0} \cdot \boldsymbol{n} d s \quad \forall \widetilde{\boldsymbol{v}}_{k+1}^{0} \in \widetilde{\mathbb{R T}}_{k+1}^{0}(K) .
$$

We have the following result.

Proposition 5.1 The Lagrange multiplier component of the solution of the defect correction DMH problem in Step S2 is the unique solution of

$$
a_{h}\left(\widetilde{\lambda}_{k+1}, \widetilde{\eta}\right)=b_{h}(\widetilde{\eta}) \quad \forall \widetilde{\eta} \in \widetilde{L}_{k+1}\left(\mathcal{E}_{h, i}\right)
$$

where

$$
\begin{aligned}
& a_{h}(\widetilde{\zeta}, \widetilde{\eta})=a_{h}\left(\widetilde{\boldsymbol{q}}_{\widetilde{\zeta}}, \widetilde{\boldsymbol{q}}_{\tilde{\eta}}\right), \\
& b_{h}(\widetilde{\eta}) \quad=\int_{\Gamma} g_{D} \widetilde{\boldsymbol{q}}_{\tilde{\eta}} \cdot \boldsymbol{n} d s .
\end{aligned}
$$

Proof. The proof is analogous to [9, Lemma 2.2]. The uniqueness of the solution of (36) is a direct consequence of the properties of problem (33).

The matrix $E$ and the right-hand side $H$ of (13) turn out to be the stiffness matrix associated with the bilinear form $a_{h}(\cdot, \cdot)$ and the linear form $b_{h}(\cdot)$ of (37), respectively.

Remark 5.1 When a Neumann part of the boundary exists, that is $\boldsymbol{q} \cdot \boldsymbol{n}_{\Gamma}=j_{N}$ on $\Gamma_{N}$, with $j_{N} \in H^{-1 / 2}\left(\Gamma_{N}\right)$ and $\Gamma=\Gamma_{D} \cup \Gamma_{N}$, such a condition is weakly enforced in the DMH method by adding corresponding terms at the right-hand side of $(9)_{3}$, this simply reflecting into an additional contribution to the righthand side of (13).

\section{A Hierarchical A-Posteriori Error Estimator}

The completion concept expressed in the representation (14) can be reinterpreted as an instance of Variational Multiscale Modeling [18], recently applied to the Discontinuous Petrov-Galerkin setting in [3], and of Enrichment Methods [12]. This reinterpretation allows to devise an explicitly computable a-posteriori hierarchical estimator for the discretization errors $\boldsymbol{q}-\boldsymbol{q}_{k}$ and $u-u_{k}$. Following [18], we assume that a decomposition of the exact solution $u, \boldsymbol{q}$ in terms of coarse and fine scales can be performed in the same spirit as 
in (14), in such a way that

$$
u:=\bar{u}+u_{u n r}, \quad \boldsymbol{q}:=\overline{\boldsymbol{q}}+\boldsymbol{q}_{u n r},
$$

where $\overline{(\cdot)}$ and $(\cdot)_{\text {unr }}$ denote the resolved and unresolved parts of the solution, respectively. According to the relative weight of coarse and fine scales, the quantities $u_{u n r}$ and $\boldsymbol{q}_{u n r}$ provide important information on the local behavior of the exact solution $u, \boldsymbol{q}$. In practice, the resolved part of the solution is approximated here by $u_{k}, \boldsymbol{q}_{k}$, while the unresolved part of the solution is dealt with by introducing for all $K \in \mathcal{T}_{h}$ the following saturation assumption

$$
\begin{aligned}
& \left\|u-u_{k+1}\right\|_{0 ; K} \leq \beta_{u}\left\|u-u_{k}\right\|_{0 ; K} \\
& \left.\left\|\boldsymbol{q}-\boldsymbol{q}_{k+1}\right\|\right|_{\text {div } ; K} \leq \beta_{q}\left\|\boldsymbol{q}-\boldsymbol{q}_{k}\right\| \|_{\text {div } ;}
\end{aligned}
$$

where $0 \leq \beta_{u}, \beta_{q}<1$. If we thus replace $u_{u n r}$ and $\boldsymbol{q}_{u n r}$ with their approximations $\widetilde{u}_{k}$ and $\widetilde{\boldsymbol{q}}_{k}$, it is immediate to check that assumption $(\mathrm{H})$ implies the following upper and lower bound for the local error

$$
\begin{aligned}
& \frac{1}{1+\beta_{u}}\left\|u_{k+1}-u_{k}\right\|_{0 ; K} \leq\left\|u-u_{k}\right\|_{0 ; K} \leq \frac{1}{1-\beta_{u}}\left\|u_{k+1}-u_{k}\right\|_{0 ; K}, \\
& \frac{1}{1+\beta_{q}}\left\|\boldsymbol{q}_{k+1}-\boldsymbol{q}_{k} \mid\right\|_{\text {div } ; K} \leq\|\| \boldsymbol{q}-\boldsymbol{q}_{k}\left\|_{\text {div } ; K} \leq \frac{1}{1-\beta_{q}}\right\|\left\|\boldsymbol{q}_{k+1}-\boldsymbol{q}_{k}\right\| \|_{\text {div } ; K} .
\end{aligned}
$$

The above relations provide the mathematical foundation for the following simple error indicator $\eta_{H}$ to drive the grid adaptation process

$$
\begin{aligned}
& \eta_{H}^{2}:=\sum_{K \in \mathcal{T}_{h}} \eta_{H, K}^{2}, \\
& \eta_{H, K}^{2}=\left\|\widetilde{u}_{k+1}\right\|_{0 ; K}^{2}+\left.\left\||| \widetilde{\boldsymbol{q}}_{k+1}\right\|\right|_{\text {div } ; K} ^{2} .
\end{aligned}
$$

Remark 6.1 The present hierarchical a-posteriori error estimator for the $D M H$ formulation is applicable to an RT approximation of arbitrary degree $k$ and is connected with the hierarchical estimators for DM methods proposed in [1] and, in particular, to the estimator $\eta_{L}$ of [24, Eq.(2.15)].

We provide a validation of the proposed a posteriori error estimator (38) for the solution of problem (9), with $\kappa=1, \sigma=0$ on $\Omega=(0,1)^{2}$ and $f$ such that the exact solution is

$$
\begin{aligned}
u=x y(x-1)(y-1) & \left(\mathrm{e}^{-100\left((x-0.5)^{2}+(y-0.1)^{2}\right)}+\mathrm{e}^{-100\left((x-0.5)^{2}+(y-0.9)^{2}\right)}\right. \\
& \left.+\mathrm{e}^{-100\left((x-0.1)^{2}+(y-0.5)^{2}\right)}+\mathrm{e}^{-100\left((x-0.9)^{2}+(y-0.5)^{2}\right)}\right) .
\end{aligned}
$$

The solution has four distinct peaks, each approximately equal to 1 , localized respectively at $(0.5,0.1),(0.5,0.9),(0.1,0.5)$ and $(0.9,0.5)$. Starting from the 

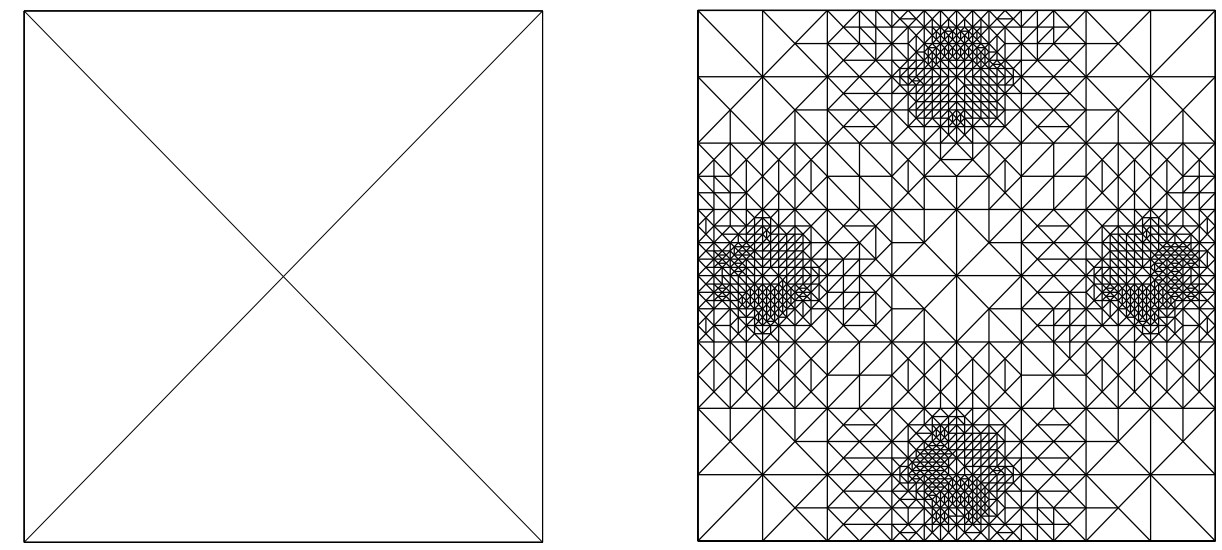

Figure 6. Left: initial triangulation $\mathcal{T}_{0}$. Right: triangulation $\mathcal{T}_{9}$.

coarse triangulation $\mathcal{T}_{0}$ shown in Fig. 6 (left) and given a tolerance tol, the refinement process is executed according to the following algorithm, for $\ell \geq 0$ :

- Step A1. Solve the discrete problem (9) on the mesh $\mathcal{T}_{\ell}$ using the fixed point iteration described in Sect. 4.2.

- Step A2. Compute $\eta_{H}^{(\ell)}$ using (38), corresponding to the mesh $\mathcal{T}_{\ell}$. Then, terminate the algorithm if

$$
\eta_{H}^{(\ell)} \leq \operatorname{tol} \eta_{H}^{(0)}
$$

otherwise go to Step A3 of the algorithm.

- Step A3. Mark the element $K$ for further refinement if $\eta_{H, K}^{(\ell)} \geq \frac{1}{2} \max _{K^{\prime} \in \mathcal{T}_{\ell}} \eta_{H, K^{\prime}}^{(\ell)}$.

- Step A4. Perform red-green-blue refinement on all the marked elements and run a closure algorithm to avoid hanging nodes.

- Step A5. Generate the new mesh $\mathcal{T}_{\ell+1}$, set $\ell:=\ell+1$, and go to Step A1.

Fig. 6 (right) shows the mesh obtained after 9 refinement steps. Notice the presence of highly refined zones corresponding to the localized peaks of the solution. Fig. 7 shows the contour lines of the computed scalar field superposed to the flux vector field on mesh $\mathcal{T}_{9}$ (left) and the convergence history for the error estimator (dashed line) and for the discretization error measured in the graph norm for $H(\operatorname{div} ; \Omega) \times L^{2}(\Omega)$ (solid line) as a function of the average mesh size (right). The results indicate that the error estimator is reliable and accurate, as the mesh size goes to zero. We observe that, in the limit of a very refined discretization, the fine scales eventually disappear, in agreement with the fact that in such a case all scales are coarse, i.e. visible. The reasoning explains why a progressively reduced number of iterations is required for the fixed-point procedure to converge as the grid becomes more refined. 



Figure 7. Left: contour lines of $u_{h}$ and field plot of $\boldsymbol{q}_{h}$ on mesh $\mathcal{T}_{9}$. Right: convergence history for the error estimator (dashed line) and the discretization error (solid line) as a function of the average mesh size.

\section{Conclusions and Future Perspectives}

In this article, we have proposed a computational procedure for the efficient implementation of Dual-Mixed Hybridized methods of arbitrary degree.

This procedure relies on three main mathematical tools: $i$ ) the use of hierarchical finite element bases which yields a vertical splitting of each approximation space into a lower degree and a defect correction part (completion); ii) the use of the Helmholtz decomposition principle for the vector-valued variable which yields an horizontal splitting of the defect correction into solenoidal and non-solenoidal subspaces; iii) the use of a fixed-point iteration to solve the approximate decoupled local subproblems stemming from $i$ ) and $i i$ ). The resulting scheme can be implemented by solving global condensed problems or local problems of reduced size. In doing this, one can benefit from the fact that the associated coefficient matrices can be factorized once for all during the iteration.

Exploiting the equivalence between hierarchical completion and Variational Multiscale Modeling, we have also devised and numerically validated a hierarchical a-posteriori error estimator for Dual Mixed methods in hybridized form. Again, the overall efficiency of the algorithm benefits from the fact that the computation of the error estimator is a by-product of the iteration procedure.

Further research activity will be devoted to:

- a deeper analysis of the connection between the present formulation and Variational Multiscale Theory, to deal with problems including possibly 
dominating advective terms;

- an application of the completion-based DMH method in the framework of variable degree formulations [10].

\section{References}

[1] B. Achchab, A. Agouzal, J. Baranger, and J.F. Maitre, Estimateur d'erreur a posteriori hiérarchique. Application aux éléments finis mixtes, Numer. Math. 80 (1998), 159-179.

[2] D.N. Arnold and F. Brezzi, Mixed and Nonconforming Finite Element Methods: Implementation, Postprocessing and Error Estimates, Math. Modeling and Numer. Anal. 19-1 (1985), 7-32.

[3] C. L. Bottasso, S. Micheletti, and R. Sacco, A multiscale formulation of the Discontinuous Petrov-Galerkin method for advective-diffusion problems, Comput. Methods Appl. Mech. Engrg. 194 (25-26) (2005), 2819-2838.

[4] F. Brezzi and M. Fortin, Mixed and hybrid finite element methods, Springer Verlag, New York, 1991.

[5] C. Carstensen, A unifying theory of a posteriori finite element error control, Numer. Math. 100 (4) (2005), 617-637.

[6] C. Carstensen and R. Hoppe, Convergence analysis of an adaptive nonconforming finite element method, Numer. Math. 103 (2) (2006), 251-266.

[7] P. Causin and R. Sacco, A Discontinuous Petrov-Galerkin method with Lagrangian multipliers for second order elliptic problems, SIAM J. Numer. Anal. 43 (1) (2005), 280-302.

[8] P.G. Ciarlet, The Finite Element Method for Elliptic Problems, North Holland, Amsterdam, 1978.

[9] B. Cockburn and J. Gopalakrishnan, A characterization of hybridized mixed methods for second order elliptic problems, SIAM Jour. Numer. Anal. 42 (1) (2003), 283-301.

[10] _ Error analysis of variable degree mixed methods for elliptic problems via hybridization, Math. Comp. 74 (252) (2005), 1653-1677.

[11] M. Crouzeix and P.A. Raviart, Conforming and non-conforming finite element methods for solving the stationary Stokes equations, R.A.I.R.O. R-3 (1973), $33-76$.

[12] C. Farhat, I. Harari, and U. Hetmaniuk, The discontinuous enrichment method for multiscale analysis, Comput. Methods Appl. Mech. Engrg. 192 (28-30) (2003), 3195-3209. 
[13] V. Girault and P.A. Raviart, Finite Element Approximation of the NavierStokes Equations, Lecture Notes in Mathematics, vol. 749, Springer Verlag, Berlin, New Jersey, 1979.

[14] J. Gopalakrishnan, A Schwarz preconditioner for a hybridized mixed method, Comp. Meth. Appl. Math. 3 (1) (2003), 116-134.

[15] R.H.W. Hoppe and B. Wohlmuth, Efficient numerical solution of mixed finite element discretizations by adaptive multilevel methods, Appl. Math. 40 (1995), $227-248$.

[16] _ Adaptive multilevel techniques for mixed finite element discretizations of elliptic boundary value problems, SIAM J. Numer. Anal. 34 (4) (1997), 1658-.

[17] _ Hierarchical basis error estimators for Raviart-Thomas discretizations of arbitrary orders, Proc. Conf. Finite Element Methods: Superconvergence, Post-processing and A Posteriori Estimates (P. Neittaanmki, ed.), Jyväskylä, Finland, Marcel Dekker, New York, 1997, pp. 155-167.

[18] T.J.R. Hughes, G.R. Feijoó, L. Mazzei, and J.B. Quincy, The variational multiscale method: a paradigm for computational mechanics, Comput. Methods Appl. Mech. Engrg. 166 (1998), 3-24.

[19] J.C. Nédélec, Notion sur les techniques d'éléments finis, Mathématiques et Applications, vol. 7, Ellipses, 1991.

[20] A. Quarteroni and A. Valli, Numerical Approximation of Partial Differential Equations, Springer-Verlag, New York, Berlin, 1994.

[21] P.A. Raviart and J.M. Thomas, A mixed finite element method for second order elliptic problems, Mathematical Aspects of Finite Element Methods, Vol. I (I. Galligani and E. Magenes, eds.), Springer-Verlag, Berlin, 1977.

[22] _ Primal hybrid finite element methods for 2nd order elliptic equations, Math. Comp. 31-138 (1977), 391-413.

[23] B. Szabó and I. Babuška, Introduction to the finite element analysis, John Wiley and Sons, New York, 1989.

[24] B.I. Wohlmuth and R.H.W. Hoppe, A comparison of a posteriori error estimators for mixed finite element discretizations by Raviart-Thomas elements, Math. Comp. 68(228) (1999), 1347-1378. 